\title{
AS CONTRIBUIÇÕES DA TERAPIA COGNITIVO COMPORTAMENTAL NA PSICOLOGIA JURÍDICA: VARAS DE FAMÍLIA
}

\author{
Kalma Souza de Mesquita ${ }^{\mathrm{T}}$ \\ Gustavo Luis Caribé Cerqueira ${ }^{2}$
}

RESUMO: Este artigo teve como objetivo geral, em revisão narrativa, em corte transversal, investıgar e mapear as contribuições da terapia cognitivo comportamental na psicologia jurídica - varas de família. Bem como, verificar a viabilidade da aplicação da Terapia Cognitivo Comportamental na psicologia jurídica varas de família; analisar as etapas da conduta psicológica, frente às demandas na psicologia jurídica nas varas de família; e, observar o andamento técnico na tomada de decisões. Foi utilizado procedimento (revisão ou levantamento) bibliográfico para elaboração e desenvolvimento dessa obra. Teve como assuntos analisados a Terapia Cognitivo Comportamental, a Psicologia jurídica, as varas de família, a Alienação Parental e as principais Técnicas científicas de coleta de dados. O objetivo proposto foi alcançado satisfatoriamente, haja vista, a psicologia jurídica como um todo e sua pretensão principal, auxiliar o melhor exercício do direito e o seu compromisso com o protagonismo humano em diferentes contextos que ela beneficia. Tudo são trocas de esclarecimentos e saberes, uma vez que, objetiva contribuir e corresponder, humanitariamente, à questão do bem-estar familiar e social.

Palavras-Chave: Psicologia jurídica. Terapia Cognitivo Comportamental. Varas de Família. Alienação Parental.

ABSTRACT: This article aimed, in a narrative review, in cross-section, to investigate and map the contributions of cognitive behavioral therapy in legal psychology - family courts. As well as, verify the feasibility of applying Cognitive Behavioral Therapy in legal psychology - family courts; to analyze the stages of psychological conduct, facing the demands in legal psychology in family courts; and, observe the technical progress in decision making. A bibliographic procedure (review or survey) was used for the elaboration and development of this work. The subjects analyzed were Cognitive Behavioral Therapy, Legal Psychology, Family Courts, Parental Alienation and the main scientific techniques of data collection. The proposed objective was satisfactorily achieved, given the legal psychology as a whole and its main intention, to help the best exercise of law and its commitment to human protagonism in different contexts that it benefits. Everything is an exchange of clarification and knowledge, since it aims to contribute and respond, humanely, to the issue of family and social well-being.

Keywords: Legal Psychology. Cognitive behavioral therapy. Family Courts. Parental Alienation.

\footnotetext{
'Graduanda em Psicologia pela FADBA E-mail: kalma.mesquita@gmail.com.

${ }^{2}$ Professor na Universidade Católica do Salvador (UCSAL) no departamento de Psicologia, é professor/orientador no curso de pós-graduação de Neuropsicologia, Terapia Cognitivo Comportamental e Saúde Mental e dependência química. Psicólogo, mestrado em Ciências da Saúde (UFBA), psicoterapeuta Cognitivo Comportamental (IWP) e Neuropsicólogo (UFBA). Especialista em Saúde Mental, Dependência Química (UFBA) e em Atenção Integral aos Usuários de Substâncias Psicoativas (UFBA). Com formação em Terapia do Esquema (IWP), Terapia Cognitivo Comportamental (IWP) e Terapia Cognitiva Processual (UFBA). Membro do Laboratório de Pesquisa em Neuropsicologia Clínica e Cognitiva (Neuroclic - UFBA), membro do International Center for Science in Drug Policy, pesquisador da Rede de Pesquisa sobre Drogas da Secretaria Nacional de Políticas sobre Álcool e outras Drogas (SENAD/MJ). Membro da Associação Brasileira de Estudos Sobre Substâncias Psicoativas (ABESUP). Com experiência em gestão pública em saúde mental e gestão acadêmica em curso de graduação (Psicologia) e curso técnico (PRONATEC de Reabilitação de Dependentes Químicos).
} 


\title{
REVISÃO DE LITERATURA
}

Ao ter em vista o objeto de estudo em comum: o homem, em sua complexidade e conflitos, a Psicologia jurídica, ou psicologia aplicada ao direito, se trata de um ramo da Psicologia que pretende auxiliar o melhor exercício do magistrado. (AMORIM, 2015).

A partir do conhecimento de que buscará em cada caso o maior beneficio para cada criança e para cada adolescente, juntamente com a equipe multidisciplinar para melhores informações e trocas de saberes, trazendo para os autos do processo a realidade psicológica das partes, e o real comportamento dos pais ou responsáveis que foram devidamente avaliados pelo psicólogo, contribuindo na decisão judicial final, o psicólogo jurídico na vara da família utiliza de sua capacidade profissional, detectando mensagens inconscientes, subjetivas entre os sujeitos, bem como causas influentes no comportamento do ser humano como um todo. (RAMOS, 2015).

Nesta área, o psicólogo coloca seus conhecimentos à disposição dos operadores do direito, assessorando-os nas ações judiciais, trazendo uma realidade psicológica das partes envolvidas. A Psicologia Jurídica enquanto aplicação do saber psicológico às práticas jurídicas envolve a Psicologia Criminal, Forense e Judiciária. Colabora ainda com o planejamento e execuções das políticas de cidadania, Direitos Humanos e prevenção de violência, com formulação, revisão e interpretação das leis. (COSTA ; LÔBO, 2013).

\begin{abstract}
Os estudos aplicados foram se organizando em áreas de interesse, e por esse motivo hoje se fala em Psicologia Comunitária, Psicologia Escolar e Educacional, Psicologia Hospitalar, Psicologia Organizacional, Psicologia do Esporte e, da mesma forma, Psicologia Jurídica e Forense. E apesar de a prática psicológica ter adquirido fama pela prática clínica, ela tem beneficiado diferentes contextos como instituições comunitárias, escolas, hospitais, empresas, ambientes esportivos, ambientes jurídicos, entre outros. (AMORIM, 2015).
\end{abstract}

A prática mostra que, perpassando pela assessoria jurídica, há o trabalho clínico cuja função terapêutica de cuidado, escuta ativa, genuinidade e empatia, facilita no acolhimento e na adesão às orientações. as intercessões entre duas áreas distintas da psicologia: jurídica e clínica cognitiva-comportamental dão-se através do: I) processo diretivo e semiestruturado, 2) orientado à resolução de problemas, que requer 3) foco e avaliação contextualizada dos problemas e das metas para sua resolução; dessa forma, os beneficiados têm suas visões ampliadas e entendem a necessidade de envolverem-se ativamente no processo intervenção. (COSTA ; LÔBO, 2013).

Os conflitos envolvendo relacionamentos familiares, quando resolvidos e encarados de maneira saudável, influenciam em toda a vida dos indivíduos envolvidos, especialmente dos filhos crianças e adolescentes, isso explica porque o Direito da família, 


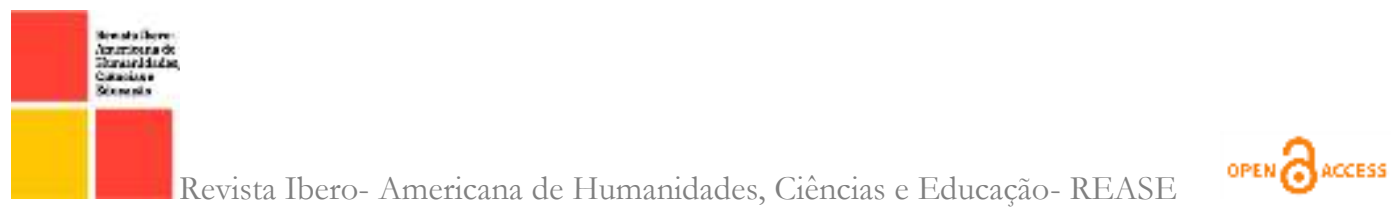

da Criança e do Adolescente são as áreas em que a Psicologia costuma receber atenção especial por parte dos órgãos jurídicos. Outrossim, é especialmente aplicável o uso de técnicas de solução de conflitos alternativas, como a mediação, onde o psicólogo pode facilitar a comunicação, a cooperar para estabelecer limites e probabilidades de cada parte e as consequências de uma decisão, nos casos em que o estado emocional costuma estar exacerbado. (AMORIM, 2015).

Os procedimentos interventivos de maior sucesso focam diversos fatores, de ordem econômica, biológica, ontológica, genética e cultural. $\mathrm{Na}$ mesma direção tratamentos multidisciplinares têm obtido melhores resultados quando comparados aos unidisciplinares; e, tem ocorrido intensificado interesse do individual no casal, bem como da capacidade dos cônjuges de se influenciarem, um ao outro. (BOLSONI-SILVA, 2009).

A literatura estrangeira têm consistentemente apontado que a terapia cognitivocomportamental oferece um modelo eficaz no tratamento de casais em desarmonia. $\mathrm{O}$ número de publicações sobre o assunto tem aumentado significativamente, na América do Norte, em evidência. Outrossim, em nossa sociedade, um número expressivo de casais enfrenta problemas decorrentes de relações disfuncionais e conflituosas. Sabe-se que tais dificuldades podem influenciar no desenvolvimento e manutenção de transtornos psíquicos, como a ansiedade e a depressão. (PEÇANHA ; RANGÉ, 2008).

As estratégias que os cônjuges utilizam para resolver seus conflitos predizem se o
desfecho da situação será efetivo ou não. Essas estratégias são comportamentos
adotados com a finalidade de encontrar uma solução para o problema, pressupõem
a necessidade de negociação entre os parceiros e são consideradas construtivas ou
destrutivas; as construtivas envolvem comunicação respeitosa, flexibilidade,
percepção do conflito como oportunidade de melhorar o relacionamento, clareza
sobre a corresponsabilidade dos cônjuges, autocontrole, tolerância e a busca
conjunta por uma solução satisfatória para o casal; enquanto as destrutivas,
caracterizam-se pela identificação de culpados, foco demasiado no problema,
comportamentos de esquiva e de retraimento, racionalização, hostilidade,
reclamações, rigidez e negatividade. Com efeito, conflitos conjugais vêm sendo
investigados na literatura internacional há cerca de cinco décadas, abrangendo
seus motivos, intensidade, freqüência e forma de encaminhamento. (COSTA, et
al, 2oi7).

A modalidade da terapia de Beck consiste na modificação dos pensamentos disfuncionais, que determinam o estado de humor, o afeto e o comportamento dos indivíduos, vem sendo utilizada em vários tratamentos de diferentes problemas, inclusive nas questões de casais em desalinho, em que a abordagem comportamental também teve 
grande influência no desenvolvimento de técnicas utilizadas (PEÇANHA; RANGÉ, 2008).

Os sentimentos de culpa e de abandono ocorrem, em pensamento, na criança, gerando uma série de circunstâncias que podem influenciar o seu desenvolvimento biopsicossocial. Pois, quando há uma dissolução dos vínculos conjugais, na maioria dos casos, as crianças começam a entrar em conflitos, sentem-se inseguras como consequência da indecisão de quem ficará como seu guardião; uma vez que, o processo de divórcio nem sempre é uma ação simples, em grande parte dos casos, compreende a toda família e, principalmente, os filhos. (FORMIGA, 2015).

A partir da revisão apresentada pode-se concluir: (a) é importante treinar comportamentos envolvidos na comunicação conjugal, expressão de sentimentos, resolução de problemas, de forma a aumentar a coesão conjugal, satisfação e empatia e reduzir o estresse; (b) diversas abordagens tem sido utilizadas em intervenções com casais e, dentre elas, a comportamental tem se mostrado efetiva a partir da literatura internacional e no Brasil há o registro de apenas um procedimento, indicando a necessidade de novos estudos; (c) o procedimento pode ser estruturado, mas precisa garantir flexibilidade as demandas individuais de cada cônjuge; (d) outras variáveis também são importantes e referem-se a interação terapêutica, seja as do cliente, seja as do terapeuta; (d) o tempo e o número de sessões utilizados em programas efetivos variaram de 4 até 30 encontros, de duas até quatro horas cada. (BOLSONI \& SILVA, 2009).

Os experimentos comportamentais, em que o paciente é incentivado a modificar as contingências de seu próprio ambiente, são importantes técnicas avaliativas, pois testam diretamente a validade dos pensamentos. A terapia cognitiva é baseada nos problemas do cliente e no estabelecimento de metas específicas, através das quais são identificados os pensamentos automáticos testáveis que impedem a realização dessas metas. A validade desses pensamentos é avaliada em conjunto por terapeuta e cliente. As técnicas comportamentais são empregadas, sobretudo, para que o paciente altere algum comportamento de seu repertório e possa, com isso, reexaminar as crenças sobre si mesmo e sobre os eventos, obter evidências factuais para suas conclusões e reformular suas avaliações. (SOUZA; CÂNDIDO, 2009).

Fazer os cônjuges e os membros da família treinarem todos os três tipos de reações - não assertiva, assertiva, agressiva - uns com os outros pode ser útil para ajudá-los a 
entender que o comportamento assertivo os beneficia, fazendo-os ter uma relação saudável. Permitir que os cônjuges observem modelos do mesmo gênero dentro da sua faixa etária relativa é muito útil para lhes mostrar a diferença entre o comportamento assertivo e os comportamentos não assertivos ou agressivos. $O$ terapeuta pode usar dramatização durante as sessões, encaminhar os indivíduos para programas de treinamento ou determinar que assistam a vídeos de treinamento da assertividade, particularmente aqueles que envolvem o contexto relacional de um casal ou de uma família. (DATILLIO, 20II).

De modo geral, as pesquisas em psicoterapia têm como objetivo identificar, testar, desenvolver e, sobretudo, estimular o uso e a disseminação de técnicas psicoterápicas válidas cientificamente visando a saúde mental dos indivíduos. Esses estudos avaliam se as psicoterapias produzem resultados efetivos, quais fatores e de que forma favorecem a remissão de sintomas e; também possibilitam o desenvolvimento fundamentado da prática profissional a demonstrar que pesquisa e clínica são, acima de tudo, indissolúveis e complementares. (COSTA, et al. 2oII).

Escutar as narrativas das histórias de vida longitudinais e transversais das

famílias desloca a postura investigativa dos psicólogos dos fatos reais. O que está em questão aqui não é a dissolução dos conflitos familiares, mas a possibilidade de uma nova reorganização desse sistema, em que não mais ocorra a devastação, que estava em curso, da subjetividade da criança. Assim, o que se investiga e se instiga ao mesmo tempo, na verdade, é a potencialidade que os sujeitos envolvidos nos conflitos judiciais teriam para criarem novos sentidos acerca do próprio material narrativo que foi apresentado. Diante disso, essas avaliações se constituem, já como intervenções e embora pontuais muitas vezes atribuem um novo valor ao caráter destrutivo das separações conjugais (THERENSE et. al., 2017).

Entre outras situações que podem estar envolvidas nas ações, o psicólogo pode estudar aspectos relacionados a casos de separação e divórcio, síndrome de alienação parental, acusações de abuso sexual (verídicas ou não), pensão alimentícia, regulamentação de visitas, adoção, infração por parte de adolescentes e destituição do poder familiar, (AMORIM, 2015).

A Terapia Cognitivo Comportamental é muito importante para as pessoas que passam pelo divórcio, principalmente porque esquemas individuais mal -adaptativos ou disfuncionais que estavam inativos, como consequência da luta e estresse da ação, podem ser ativados. Indivíduos que experienciam baixa autoestima ou não se sentem merecedores de amor ou temem a rejeição, são um bom exemplo. A intensidade do sentimento de 
rejeição e as consequências profundas de um divórcio são capazes de criar um estado de crise. Assim, os indivíduos que experimentam a dor emocional extremam da rejeição talvez interpretem o pedido de divórcio por parte de seus parceiros como apenas mais uma medida reafirmadora do seu desvalor. Tais cidadãos podem vir a crer fortemente de que "Eu não sou digno do amor de ninguém e, portanto, jamais conseguirei permanecer casado”. (DATILLIO, 20II).

Ademais, outros procedimentos utilizados são as alterações de comportamentos que formam padrões negativamente específicos. E, se pode afirmar que os principais objetivos da terapia cognitivo-comportamental, no tratamento de casais em conflito, são a reestruturação de cognições inadequadas, o manejo das emoções, a modificação de padrões de comunicação disfuncionais e o desenvolvimento de estratégias para solução de problemas rotineiros mais efetivos. (PEÇANHA E RANGÉ, 2008).

\begin{abstract}
As crianças implicadas são postas como artigo da disputa e por meio dela se sentem culpadas pela separação e as brigas que porventura ocorrem no seio das famílias, na maioria dos casos. Dá-se então, o ofício desafiador do Psicólogo nesse contexto, que traz a marca da ambiguidade na compreensão dos fatos que vão subsidiar os ações jurídicas de disputa de guarda, pois essa compreensão envolve a subjetividade de cada criança, os conflitos nos quais os pais estão envolvidos e o que é regulamentado pelas Leis do Direito. Inobstante, tudo dependerá de como esse contexto é representado para o filho, pois cada sujeito é único e as situações também são experienciadas de formas ímpares em meio a mais complexa pluralidade. (FORMIGA, 2015).
\end{abstract}

Um instrumento comumente utilizado é a entrevista, pois através dela consegue-se obter informações relevantes sobre a história de vida do indivíduo e esses dados são importantes no entendimento das motivações que o leva à prática de determinado ato. Para a realização de avaliações citadas anteriormente, utiliza-se, entre outros o processo de testagem, uma atividade do psicólogo nas investigações com o indivíduo. Sobre esse assunto, ressalta-se a importância de verificar a confiabilidade e a validade dos instrumentos e do modelo teórico utilizado com a finalidade de verificar se respondem ao objetivo do procedimento. (SILVA e FONTANA, 20II).

A partir de reflexões e análises críticas das demandas que reduzem o fazer do psicólogo à realização de perícias, novas aberturas surgiram, de modo que os psicólogos ampliaram suas intervenções nos casos, realizando aconselhamento, encaminhamento, orientação, práticas alternativas de resolução pacífica de conflitos, mediação, participação ativa na articulação de políticas públicas de atendimento em rede, entre outros. Outrossim, destacam-se, ainda, as práticas de atendimento psicológico com as famílias, trabalho com 

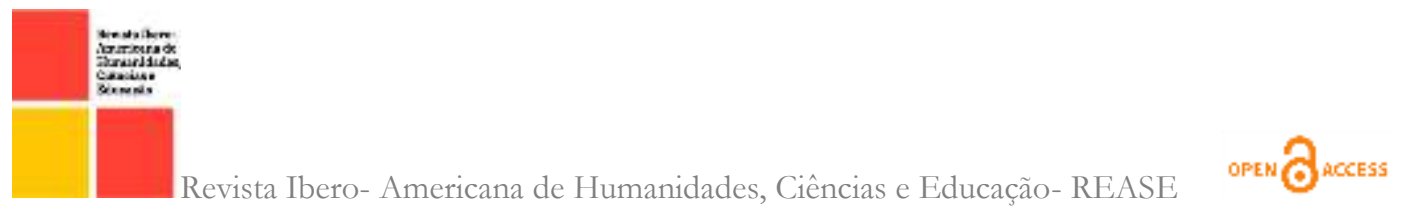

grupos, elaboração de laudos, pareceres, informes e relatórios, dentre outras possibilidades de atuação. (THERENSE, 2017).

A elaboração de documentos no processo de avaliação psicológica, comumente ligada ao contexto de perícias judiciais, envolvendo utilização de testes e desenvolvimento de escalas e outros instrumentos que avaliam vínculos parentais representa uma importante fatia da atuação do psicólogo no contexto da alienação parental. Destarte, é importante destacar que em momento nenhum se admite a possibilidade de que o psicólogo perito, que esteja realizando uma avaliação de uma família em contexto de litígio judicial, enuncie opiniões, ou "conclusões" que possam se assemelhar a "julgamentos", pois são posicionamentos repletos de juízos de valor, que se afastam completamente da psicologia, enquanto ciência e profissão. (JAGER \& RODRIGUES, 2016).

A perícia é descrita também enquanto processo de investigação, análise e comunicação. Isto é, o "instrumento científico, pertinente à especialidade da Psicologia Jurídica, que tem como objetivo maior elucidar fatos e/ou situações para auxiliar a compreensão de fatores subjetivos presentes nas relações humanas e que interferem no andamento dos processos judiciais". Este instrumento consiste em um meio de prova nos autos jurídicos. Esta prova pericial consiste em exame, vistoria ou avaliação, pondo então em xeque qualquer fragilidade que a mesma venha porventura possuir na apresentação de produtos objetivos. Em outras palavras, transcrevendo epistemologicamente os termos acima, entende-se que a perícia propõe um desvelamento (investigação), redução (análise) e formulação de enunciados de verdade para fins de divulgação dos resultados (comunicação), sendo estes validados e de caráter preferencialmente irrefutável, em que, a demanda é justificada pela própria definição do que consiste perícia psicológica. (THERENSE, et al. 2017).

No contexto das possibilidades de aplicação da abordagem cognitivo comportamental no âmbito jurídico, faz-se necessário uma boa vinculação do usuário ao serviço, através da empatia e da psicoeducação; uma vez que, a atuação objetiva intervir protetivamente junto a crianças e adolescentes com seus direitos violados embasada no Estatuto da Criança e do Adolescente. Tal prática, assim como a clínica cognitiva, se configura, inicialmente, pela conceitualização do caso, realizando avaliação histórica e do contexto de vida atual, com vistas a identificar as vulnerabilidades específicas, os fatores de risco e delinear a estratégia de intervenção que diminua a situação de vulnerabilidade. Nesse sentido, procura-se facilitar a compreensão do sujeito acerca de seus problemas e de seus direitos enquanto cidadão, bem como esclarecer a função do setor psicossocial e da importância do envolvimento do mesmo no processo para solucionamento do impasse. (COSTA \& LÔBO, 2013). 
Concernente aos envolvidos no litígio, utiliza-se a Psicoeducação, pois, é um momento em que as tensões relacionadas ao fato dos interessados estarem em um ambiente judiciário podem ser amenizadas, viabilizando maior estabilidade emocional no momento da audiência; uma vez que, ela fornece informação e suporte, ou uma combinação destes, educando a respeito de determinado contexto e, mostra-se efetivo em atingir as necessidades das famílias, orientando de forma preventiva quanto às necessidades dos interessados. (THERENSE, et al. 2017).

Trazendo para os autos do processo a realidade psicológica das partes, e o real comportamento dos pais ou responsáveis que foram devidamente avaliados pelo psicólogo, o psicólogo jurídico na vara da família utiliza de sua capacidade profissional contribuindo na decisão judicial final, detectando mensagens subjetivas entre os sujeitos, mensagens inconscientes, bem como causas influentes no comportamento do ser humano como um todo. Dessa maneira, buscará, juntamente com a equipe multidisciplinar em cada caso, o maior beneficio para cada criança e para cada adolescente, a partir desse conhecimento, para melhores informações e trocas de saberes. (RAMOS, 2015).

\section{REFERÊNCIAS}

AMORIM, S. M. A psicologia e suas contribuições nas práticas jurídicas. Revista Vox. Revista da Faculdade de Direito e Ciências Sociais do Leste de Minas - Reduto/MG, p.7o. Edição n.I, vol. I, 2015 .

BOLSONI-SILVA, A. T. Intervenção comportamental para casais: um estudo de caso. Revista Psicologia. P. 05. Ano 2, Número I. Volume 2. Ribeirão Preto - SP, 2009.

COSTA, C. B, et al. Terapia de Casal e Estratégias de Resolução de Conflito: Uma Revisão Sistemática. Psicologia: Ciência e Profissão. 208-223. v. 37, nº 2017.

COsTA, J. M. F. S. ; LÔBO, L. T. A. Abordagem Cognitivo Comportamental e Psicologia Jurídica: Intercessão possível? Promotoria da Infância e Juventude - Ministério Público, Recife $2013 . \quad \mathrm{PE}), \quad$ Disponível em: http://cbtc.fbtc.org.br/Edicao/2013/trabalhosCBTC/P-IAoo8.pdf. Acesso em: 31.o8.ig.

DATTILIO, F. M. Manual de terapia cognitivo-comportamental para casais e famílias. P. 197. Porto Alegre : Artmed, 2011.

FORMIGA, N. S., at al. A prática do psicólogo no atendimento a crianças envolvidas em litígio de guarda. Estação Científica - Juiz de Fora, no 13, janeiro - junho / 2015. 
JAGER, M. E. ; RODRIGUES J. G. Atuação do psicólogo no contexto da alienação parental: uma revisão sistemática da literatura nacional. Multiciência Online. P. 9. Universidade Regional Integrada do Alto Uruguai e das Missões - Campus Santiago. 2016.

PEÇANHA, R. F.; RANGÉ, B. P. Terapia cognitivo-comportamental com casais: uma revisão. Revista Brasileira de Terapias Cognitivas, pp. o8- 09, Volume 4, Número I, 2008.

RAMOS, N. F. B. A Atuação E Relevância Do Psicólogo Jurídico Nas Varas De Família Do Fórum Tj/Al De Maceió/Al. Ciências humanas e sociais. Maceió, v. 3 , n.I . P. I8o. Novembro, 2015. Disponível em: periodicos.set.edu.br. Acesso em: 21.10.19.

SILVA, M. C. R; FONTANA, E. Psicologia Jurídica: Caracterização da Prática e Instrumentos Utilizados. Estudos Interdisciplinares em Psicologia. Artigo, vol.2, no.I. Londrina, 20II. Disponível em: http://pepsic.bvsalud.org/scielo.php? \&pid=S2236-64072011000100005. Acesso em: 08.02.2020.

SOUZA, I. C. W.; CÂNDIDO, C. F. G. Diagnóstico psicológico e terapia cognitiva: considerações atuais. Revista Brasileira de Terapias Cognitivas, p. 90. Volume 5, Número 2, 2009. Disponível em: http://pepsic.bvsalud.org/scielo.php?script=sci_arttext\&pid=SI808-56872009. Acesso em: 25.09.19.

THERENSE, M., et al. Psicologia Jurídica e Direito de Família: Para além da perícia psicológica. Pp. 07, 52, 255. UEA Edições: Manaus, 2017. Disponível em: http://newpsi.bvs_direito_familia.pdf. Acesso em: 13.ro.19. 